\title{
Perilla extract potentiates efferocytosis by macrophages: Implications for resolution of inflammation
}

\author{
Seung Hyeon Kim ${ }^{\mathrm{a}, \mathrm{b}}$, Deung Dae Park ${ }^{\mathrm{a}}$, Wonki Kim ${ }^{\mathrm{a}}$, Takuma Shio ${ }^{\mathrm{d}}$, \\ Jun Takanari ${ }^{\mathrm{d}}$, Takehito Miura ${ }^{\mathrm{d}}$ and Young-Joon Surh ${ }^{\mathrm{a}, \mathrm{b}, \mathrm{c}^{*}}$
}

\begin{abstract}
aTumor Microenvironment Global Core Research Center, College of Pharmacy, Seoul National University, Seoul, South Korea
${ }^{\mathrm{b}}$ Cancer Research Institute, Seoul National University, Seoul, South Korea

${ }^{\mathrm{c}}$ Department of Molecular Medicine and Biopharmaceutical Sciences, Graduate School of Convergence Science and Technology, Seoul

National University, Seoul, South Korea

dAmino Up Co., Ltd., Sapporo, Japan

${ }^{*}$ Corresponding author: Young-Joon Surh, College of Pharmacy, Seoul National University, Seoul, South Korea. Tel: +82 2 880 7845; Fax:

+82 883 2906; E-mail: surh@snu.ac.kr
\end{abstract}

DOI: $10.31665 /$ JFB.2020.10231

Received: June 22, 2020; Revised received \& accepted: June 24, 2020

Citation: Kim, S.H., Park, D.D., Kim, W., Shio, T., Takanari, J., Miura, T., and Surh, Y.-J. (2020). Perilla extract potentiates efferocytosis by macrophages: Implications for resolution of inflammation. J. Food Bioact. 10: 86-95.

\begin{abstract}
Perilla frutescens (Lamiaceae) has strong anti-inflammatory as well as antioxidant, antimicrobial, anti-allergic, antidepressant, anticancer, and neuroprotective effects and hence has diverse therapeutic applications. We have previously reported that oral administration of the standardized perilla extract ameliorated the experimentally induced colitis in mice by blocking the activation of two prototypic pro-inflammatory transcription factors, NF-KB and STAT3. Upon inflammatory insult, tissue resident macrophages produce vasoactive and chemotactic mediators, which increase vascular permeability and promote the infiltration of leukocytes, especially neutrophils, into the inflamed site where they eliminate or neutralize invading molecules. Neutrophils at the inflamed site undergo apoptosis, and apoptotic cells are removed by macrophages through phagocytosis, a process termed 'efferocytosis'. This terminates the inflammatory responses. If resolution of inflammation fails, inflammation response becomes excessive and prolonged which can cause tissue damage and loss of the function implicated in pathogenesis of a broad spectrum of human disorders. Here, we report that the standardized leaf extract of $P$. frutescens potentiates the efferocytic activity of macrophages in a zymosan-induced peritonitis model, which appears to be mediated via the PPARy activation. We also validated the stimulatory effects of the perilla extract on efferocytosis by isolated peritoneal and bone marrow derived macrophages co-cultured with apoptotic cells.
\end{abstract}

Keywords: Perilla; Perilla frutescens; Efferocytosis; Resolution of inflammation; Rosmarinic acid.

1. Introduction

Acute inflammation is a physiological response to microbial infection and tissue injury which neutralizes the harmful organisms and agents via the host innate immune system (Lee and Surh, 2012). Upon inflammatory insults, the innate immune system alarms the circulating neutrophils, leading to their recruitment to the inflamed site. Tissue resident macrophages are key immune sentinels and thus are among the first immune cells to respond to tissue damage by producing cytokines that initiate and orchestrate the recruitment of neutrophils from the blood into the tissue (Soehnlein and Lindbom, 2010; Elliott et al., 2017). After performing their action at the inflamed site, neutrophils undergo apoptosis which is a characteristic of self-limiting inflammation upon acute injury or infection. Macrophages, and to a lesser extent by other 'professional' phagocytes (e.g., monocytes and dendritic cells) and 'non-professional' 
phagocytes (e.g., epithelial cells), ingest dead or dying neutrophils, a process termed 'efferocytosis' essential for resolution of inflammation (Boada-Romero et al., 2020).

The most obvious anti-inflammatory effect of efferocytosis is the physical sequestration of dead or dying cells to limit the release of intracellular damage-associated molecular patterns that can drive inflammation (Elliott et al., 2017). Disruption of homeostatic efferocytosis leads to accumulation of uncleared apoptotic cells, which can provoke spontaneous inflammation by releasing inflammatory contents and autoimmune disease. However, apoptotic cells are thought to be beneficial in self-resolving inflammation by helping to reprogram tissue macrophages from a pro-inflammatory to a pro-resolution state (Elliott et al., 2017). Thus, clearance of apoptotic neutrophils prompts a switch from a pro- (M1) to an antiinflammatory/proresolving (M2) macrophage phenotype which is a prerequisite for macrophage egress via the lymphatic vessels favouring return to tissue homeostasis (Ortega-Gómez et al., 2013). Clearance of apoptotic neutrophils also triggers the production of additional mediators such as transforming growth factor-beta and interleukin (IL)-10 that suppress the progression of inflammation and promote repair of damaged tissues (Barnig and Levy, 2015).

Proper termination of the inflammatory cascade is important to allow execution of tissue repair and resolution responses required to restore normal tissue function. If timely resolution of acute inflammation fails, inflammation persists and can progress to a chronic state which is a root of many human disorders (Lee et al., 2013). Resolution of inflammation is an active coordinated process regulated by distinct anti-inflammatory and pro-resolving endogenous lipid mediators, such as resolvins and lipoxins (Lee and Surh, 2012). However, their production is transient and limited, and may be overwhelmed by extensive infection or injuries. In this context, it is worthwhile searching for exogenous sources of proresolving as well as anti-inflammatory substances, especially those that have been used for long period of time in herbal medicine or for a culinary purpose, so their safety has been somehow verified.

Perilla frutescens (L.) Britton var. belongs to an annual herb of the mint family (Lamiaceae). It has been used as a valuable source of culinary and medicinal materials (Bachheti et al., 2014). P. frutescens has been used in folk medicine in the management of anxiety, depression, asthma, coughs, colds, allergies, fever, headache, stuffy nose, constipation, abdominal pain, indigestion, and intoxication (Ahmed, 2018). The leaves of $P$. frutescens have strong anti-inflammatory as well as antioxidant, antimicrobial, anti-allergic, antidepressant, anticancer, and neuroprotective effects and hence have various therapeutic applications. The ethanol extracts of $P$. frutescens leaves, could significantly suppress $\mathrm{Th} 2$ responses and airway inflammation in allergic murine model of asthma (Chen et al., 2015). The ethanol extract of perilla leaves significantly suppressed secretion of IL-5 and IL-13 from ovalbumin-stimulated splenocytes of $\mathrm{Balb} / \mathrm{c}$ mice. The inflammatory mediators, such as eotaxin and histamine, and total cells, particularly eosinophils in bronchoalveolar lavage fluid, were also reduced (Chen et al., 2015). The $P$. frutescens leaf extract has been reported to ameliorate ultraviolet radiation-induced extracellular matrix damage in hairless mouse skin which was associated with reduced epidermal skin thickness and MMP-13 expression. (Bae et al., 2017).

The protective effects of perilla against experimentally induced airway inflammation were investigated. The ethanol extract of $P$. frutescens $(100 \mathrm{mg} / \mathrm{kg})$ given orally to mice was found to inhibit tumor necrosis factor- $\alpha$ (TNF- $\alpha$ ) production in the lung, after intranasal administration of lipopolysaccharide (LPS) (Lim et al., 2014). The ethanol extract of $P$. frutescens suppressed ex- pression of pro-inflammatory cytokines (TNF- $\alpha$, IL-1 $\beta$ and IL-6), pro-inflammatory enzymes including inducible nitric oxide synthase and cyclooxygenae-2 (COX-2) and their regulator NF- $\mathrm{KB}$ in LPS-stimulated murine macrophage RAW 264.7 cells (Lee and Han, 2012). Furthermore, a monoterpenoid and an alkaloid isolated from $P$. frutescens showed a remarkable inhibitory effect on the production of the inflammatory mediator, nitric oxide (NO) and pro-inflammatory cytokines (TNF- $\alpha$ and/or IL-6) in LPS-stimulated RAW264.7 cells (Wang et al., 2018).

Perilla contains polyphenols such as rosmarinic acid, luteolin, apigenin, and caffeic acid which have a large diversity of biological activities including anti-inflammatory effects (Asif, 2012; Makino et al., 2001; Ueda and Yamazaki, 2001; Lim et al., 2014; Kwak and Ju, 2015). It was reported that a standardized perilla extract (SPE) dissolved in drinking water ameliorated dextran sulfate sodium (DSS)-induced colitis in mice by suppressing production of proinflammatory cytokines (Urushima et al., 2015). We also demonstrated that administration of SPE (20 and $100 \mathrm{mg} / \mathrm{kg}$ ) by gavage protected against DSS-induced murine colitis by blocking the activation of two prototypic proinflammatory transcription factors, NF- $\kappa$ B and STAT3 (Park et al., 2017). In addition, SPE treatment to CCD $841 \mathrm{CoN}$ human normal colon epithelial cells attenuated the TNF- $\alpha$-induced expression/ activation of mediators of proinflammatory signaling (Park et al., 2017).

In the present study, we have evaluated the proresolving potential of SPE with focus on its capability to induce efferocytosis.

\section{Materials and methods}

\subsection{Materials}

Triton X-100, zymosan A, GW9662, and rosmarinic acid were purchased from MilliporeSigma (Billerica, MA, USA). The zymosan A-FITC, F4/80 antibody, Gr-1 antibody, bovine serum albumin (BSA), and peroxisome proliferator-activated receptor gamma $(\operatorname{PPAR} \gamma)$ antibody were obtained from Thermo Fisher Scientific (Waltham, MA, USA). The aqueous extract powder of $P$. frutescens (leaves) supplied by Amino Up Co. Ltd. (Sapporo, Japan) contained rosmarinic acid, $1.098 \%$; caffeic acid, $0.136 \%$; apigenin, $0.034 \%$; apigenin $\quad 7-O$-[ $\beta$-glucuronosyl $(1 \rightarrow 2) \beta$-glucuronide $]$, $2.135 \%$; apigenin $7-O-\beta$-glucuronide, $0.104 \%$; luteolin, $0.007 \%$; luteolin $7-O$ - $[\beta$-glucuronosyl $(1 \rightarrow 2) \beta$-glucuronide], $0.632 \%$; luteolin $7-O$ - $\beta$-glucuronide, $0.062 \%$; scutellarin, $0.498 \%$; vicenin- 2 , $0.149 \%$; total flavonoid, $3.621 \%$.

\subsection{Zymosan A-induced peritonitis}

C57BL/6 (6-9 wk old) were obtained from Central Lab Animal, Inc (Seoul, South Korea). Animal experiments were approved by the Institutional Animal Care and Use Committee at Seoul National University. The mice were injected with zymosan A (30 mg $/ \mathrm{kg})$ intraperitoneally. After $12 \mathrm{~h}$, the mice were given SPE (100 mg/ $\mathrm{kg}$ ) or rosmarinic acid $(20 \mathrm{mg} / \mathrm{kg})$ by intraperitoneal injection. The mice were killed $6 \mathrm{~h}$ later. The peritoneal exudates were collected for analyses.

\subsection{In vivo efferocytosis assay}

To investigate the efferocytosis in vivo, exudate cells from mice 
peritoneum were stained with anti-F4/80-antibody (Thermo Fisher Scientific; Waltham, MA, USA), permeabilized with $0.1 \%$ Triton $\mathrm{X}-100$, and then labeled with anti-Gr-1 antibody (Thermo Fisher Scientific, Waltham, MA, USA). The macrophages engulfing apoptotic neutrophils were analyzed by flow cytometry. All samples were analyzed by using BD FACS Calibur flow cytometry and Flow Jo software.

\subsection{Isolation of peritoneal macrophages (PMs)}

Six-week-old male C57BL/6 mice were treated intraperitonally with $3 \%$ Brewer thioglycollate medium $(1 \mathrm{ml})$. After 4 days, the abdominal skin of the mice was retracted to expose the peritoneal wall and through the wall, $10 \mathrm{ml}$ of cold harvest buffer [ $3 \mathrm{mM}$ EDTA in phosphate buffered saline (PBS)] was injected. The fluid was aspirated slowly and dispensed into a $50 \mathrm{ml}$ conical tube. The peritoneal exudates were centrifuge at $400 \mathrm{~g}$ for $8 \mathrm{~min}$ at $4{ }^{\circ} \mathrm{C}$. After removing supernatant, the pellet was suspended in $200 \mu \mathrm{l} 1 \mathrm{X}$ red blood cell (RBC) lysis buffer (eBioscience) and incubated in ice for 30-60 sec. Then the cells were suspended with $4 \mathrm{ml}$ PBS and centrifuged at $400 \mathrm{~g}$ for $8 \mathrm{~min}$ at $4{ }^{\circ} \mathrm{C}$. Finally, the cells were divided into culture dishes with DMEM-F12 [with 10\% fetal bovine serum (FBS), antibiotic-antimycotic mixture, glutaMAX ${ }^{\mathrm{TM}}$ ] after cell counting.

\subsection{Preparation of bone marrow derived macrophages (BM- DMs)}

The femur and the tibia were isolated from $\mathrm{C} 57 \mathrm{BL} / 6$ mice. Bone marrow cells were harvested from the femur and tibia of C57BL/6 with cold PBS containing 2\% heat-inactivated FBS. The collected cells were then filtered through a $70-\mu \mathrm{m}$ Falcon nylon cell strainer. $\mathrm{RBCs}$ were then removed using $\mathrm{RBC}$ lysis buffer while incubating on ice for $2 \mathrm{~min}$. The remaining cells were resuspended in DMEM containing $10 \%$ heat-inactivated FBS and $20 \mathrm{ng} / \mathrm{ml} \mathrm{M-CSF}$, seeded in petri dishes and incubated at $37^{\circ} \mathrm{C}$ for 7 days. Medium containing M-CSF was changed once after 3 days of incubation. BMDMs were detached from petri dishes and used for experiments.

\subsection{Efferocytosis assay with isolated macrophages}

Thymus from C57BL/6 was ground to harvest thymocytes. To obtain apoptotic thymocytes, the cells were treated with $0.1 \mu \mathrm{M}$ dexamethasone, and then incubated at $37{ }^{\circ} \mathrm{C}$ in a humidified incubator containing $5 \% \mathrm{CO}_{2}$ for $16 \mathrm{~h}$. The apoptotic cells were labeled with $1 \mu \mathrm{lof} 1 \mathrm{mg} / \mathrm{ml}$ of a $\mathrm{pH}$ sensitive dye, pHrodo-SE for $30 \mathrm{~min}$ at room temperature and co-cultured for $1 \mathrm{~h}$ with PMs or BMDMs pretreated with SPE. BMDMs were washed to remove free apoptotic cells, labeled with anti-mouse F4/80 antibody and subjected to flow cytometry for measuring the macrophages that engulf apoptotic cells. To quench fluorescence from nonphagocytized pHrodoSE-labeled apoptotic cells, cells were washed and resuspended in basic buffer ( $\mathrm{pH} 8.8$ ), and the flow cytometry assay was conducted. Apoptotic thymocytes engulfed in PMs were visualized under a microscope without macrophage labeling.

\subsection{Phagocytosis assay}

The thioglycolate-elicited PMs were treated with SPE and they were co-incubated with zymosan A-FITC for an additional $1 \mathrm{~h}$.
The phagocytic activity of macrophages was analyzed by immunostaining using F4/80 antibody. The macrophages engulfing zymosan A-FITC were visualized using an Eclipse Ti-U inverted microscope (Nikon; Tokyo, Japan).

\subsection{Immunocytochemical analysis}

The thioglycolate-elicited PMs were treated with SPE $(50 \mu \mathrm{g} /$ $\mathrm{ml}$ ) for $4 \mathrm{~h}$. After fixation with $10 \%$ formalin solution for $30 \mathrm{~min}$ at room temperature, cells were permeabilized with $0.2 \%$ Triton $\mathrm{X}-100$, incubated with blocking agents (PBS containing 5\% BSA), washed with PBS, and incubated with a diluted (1:100) primary PPAR $\gamma$ antibody overnight at $4{ }^{\circ} \mathrm{C}$. After washing with PBS, cells were incubated with a diluted (1:100) secondary antibody. The samples were examined by an Eclipse Ti-U inverted microscope (Nikon; Tokyo, Japan).

\subsection{Flow cytometry analysis for PPARY expression}

To analyze the expression of PPAR $\gamma$ in PMs, cells were fixed with $2 \%$ formaldehyde in PBS. The permeabilization of cells was preceded with $0.2 \%$ Tween- 20 in PBS for 15 min at room temperature. The PPAR $\gamma$ antibody in PBS containing $2 \%$ BSA was applied for $1 \mathrm{~h}$ at $4{ }^{\circ} \mathrm{C}$. After washing with PBS, cells were incubated with FITC conjugated secondary antibody for $1 \mathrm{~h}$. All samples were analyzed by BD FACS Calibur flow cytometry and Flow Jo software.

\subsection{Western blot analysis}

For Western blot analysis, the total protein concentration was determined by using the bicinconinic acid protein assay kit (Pierce). Cell lysates (30-50 $\mu \mathrm{g}$ protein) were mixed and boiled in a sodium dodecyl sulfate (SDS) sample buffer for $5 \mathrm{~min}$ before 8-15\% SDS-polyacrylamide gel electrophoresis (SDS-PAGE). After separation by SDS-PAGE, the gels were transferred to a polyvinylidene difluoride (PVDF) membrane (Gelman Laboratory). The blots were blocked in $5 \%$ fat-free dry milk in Trisbuffered saline containing $0.1 \%$ Tween 20 (TBST) for $1 \mathrm{~h}$ at room temperature. The membranes were incubated for 12-24 h at $4{ }^{\circ} \mathrm{C}$ with dilutions of a primary antibody for PPAR $\gamma$ (MilliporeSigma; Billerica, MA, USA). The membranes were washed, followed by incubation with 1:4,000 dilution of respective horseradish peroxidase (HRP)-conjugated secondary antibodies (rabbit or mouse) (Zymed Laboratories) for $2 \mathrm{~h}$, and again washed with TBST. Protein expressed was visualized with an enhanced chemiluminescence detection kit (Amersham Pharmacia Biotech) and LAS-4000 image reader (Fuji film) according to the manufacturer's instructions.

\subsection{Reverse transciptation polymerase chain reaction (RT- PCR)}

Total RNA was isolated by using TRIzol ${ }^{\circledR}$ (Invitrozen) according to the manufacturer's protocol. Ten $\mu \mathrm{g}$ of total RNA was reverse transcribed with MLV reverse transcriptase at $42{ }^{\circ} \mathrm{C}$ for $50 \mathrm{~min}$ and at $72{ }^{\circ} \mathrm{C}$ for $15 \mathrm{~min}$. PCR was conducted according to the 3 -step standard procedures. Amplified products were analyzed on $2 \%$ agarose gel electrophoresis, stained with SYBR ${ }^{\circledR}$ Green (Invitrogen) and photographed using fluorescence in LAS-4000. 


\section{Results}

\subsection{Perilla extract enriched with rosmarinic acid promotes ef ferocytotic activity of macrophages in a zymosan A-induced mu- rine peritonitis model}

Considering an important role of macrophages in clearance of apoptotic cells for resolution of inflammation, we investigated the effects of SPE on ability of macrophages to engulf apoptotic neutrophils. To determine effects of SPE on efferocytotic activity of macrophages in vivo, we utilized the zymosan A-induced murine peritonitis model. SPE was injected into the mouse peritoneum at $12 \mathrm{~h}$, when the total leukocyte count peaked in the zymosan A-induced peritoneal inflammation (Kim et al., 2015). The PMs were stained with the F4/80 antibody, followed by permeabilization to label the neutrophils with the Gr-1 antibody. Six hours later, peritoneal cells with positive staining of both F4/80 (macrophage marker) and Gr-1 (neutrophil marker) were selectively detected by flow cytometry.

The proportion of macrophages engulfing apoptotic neutrophils $\left(\mathrm{F} 4 / 80^{+} \mathrm{Gr}-1^{+}\right)$is the indicative of efferocytic activity. As shown in Fig. la, mice treated with zymosan A and SPE (100 mg/kg) showed macrophage-mediated efferocytosis to a greater extent than that achieved in mice challenged with zymosan A alone as an adaptive response. One of the principal anti-inflammatory ingredients of perilla is rosmarinic acid (Makino et al., 2001). The higher proportion of macrophages engulfing apoptotic neutrophils $\left(\mathrm{F} 4 / 80^{+} \mathrm{Gr}-1^{+}\right)$was shown in the mice treated with zymosan A and rosmarinic acid $(20 \mathrm{mg} / \mathrm{kg})$ compared with the mice injected with zymosan A alone (Fig. 1b).

Phagocytosis of pathogens by macrophages is crucial for the successful resolution of inflammation induced by microbial infection. When the PMs isolated from thioglycollate-elicited mice were treated with SPE $(50 \mu \mathrm{g} / \mathrm{ml})$, their phagocytic capability was enhanced (Fig. 1c).

\subsection{Perilla extract potentiates the efferocytic activity of isolat- ed macrophages}

In the next experiment, we assessed the effects of SPE on efferocytic activity of cultured macrophages. PMs pre-treated with PE for $4 \mathrm{~h}$ were found to be more active than vehicle treated control cells in engulfing apoptotic thymocytes (red puncta) compared to control cells (Fig. 2a). Because macrophages have different properties depending on their origin, BMDMs from mice were also used to examine the SPE effects. BMDMs isolated from C57/BL6 mice were mixed with pHrodo-labeled apoptotic thymocytes and subjected to FACS and immunocytochemistry. BMDMs were stained with anti-mouse F4/80 antibody and analyzed for the proportion of F4/80+ pHrodop cells + representing the macrophages that engulfed apoptotic cells.

In SPE-treated macrophages, their ability to take up pHrodo-SElabeled apoptotic thymocytes was potentiated as determined by both flow cytometry (Fig. 2b) and immunofluorescence (Fig. 2c) analyses.

\subsection{Perilla extract inhibits the proinflammatory gene expres- sion while it induces anti-inflammaory gene expression}

To figure out whether SPE also has anti-inflammatory effects as well as proresolving activity in the macrophages, the PMs from 6-weekold female C57BL/6 mice were pre-treated with SPE for $2 \mathrm{~h}$ before LPS $(100 \mathrm{ng} / \mathrm{ml})$ treatment. The mRNA expression of inflammatory cytokines, $I L-6, I L-12, I L-23$, and $T N F-\alpha$ was increased by LPS treatment, and this was inhibited by SPE (Fig. 3a). We also determined effects of SPE on expression of anti-inflammatory cytokines. The mRNA expression of IL-10, a cordinal anti-inflammatory cytokine, was upregulated at $1 \mathrm{~h}$ and $2 \mathrm{~h}$. The expression of a prototypic antiinflammatory enzyme, $\mathrm{HO}-1$ was also increased from $2 \mathrm{~h}$ and gradually decreased (Fig. 3b). The mRNA levels of IL-10 and HO-1 were also enhanced in SPE-treated BMDMs (Fig. 3c).

\subsection{Perilla extract induces efferocytosis through upregulation of PPARy expression in PMs}

PPAR $\gamma$ in macrophages has been considered to play a key role in the efferocytosis for the resolution of inflammation (Croasdell et al., 2015; Yoon et al., 2015). PPAR $\gamma$ activation is known to prime human monocytes into anti-inflammatory M2 macrophages. This prompted us to determine PPAR $\gamma$ expression in thiogycollate-elicited PMs. The PMs from mice treated with SPE showed elevated PPAR $\gamma$ expression in the zymosan A-induced peritonitis model (Fig. 4a). The thioglycolate-elicited macrophages from mice administered with SPE $(100 \mathrm{mg} / \mathrm{kg})$ showed increased PPAR $\gamma$ expression as determined by flow cytometric (Fig. 4b) and immunoblot (Fig. 4c) analyses. We also measured the SPE-induced PPAR $\gamma$ expression in cultured PMs. The thioglycolate-elicited macrophages treated with SPE $(50 \mu \mathrm{g} / \mathrm{ml})$ showed increased PPAR $\gamma$ expression which was visualized by immunofluorescence staining (Fig. 4d). To further investigate whether SPE could stimulate efferocytosis through PPAR $\gamma$ activation, we used a PPAR $\gamma$ antagonist GW9662. The GW9662 treatment inhibited SPE-induced efferocytotic activity of macrophages in the zymosan A-induced peritoneal inflammation (Fig. 4e).

\section{Discussion}

Acute inflammation is a physiologic response to tissue injury or infection and is intrinsically beneficial as it can remove or inactivate the harmful factors. In this context, normal inflammatory response is self-limiting, as it resolves with the restoration of tissue homeostasis. If resolution of inflammation fails, inflammation response becomes excessive and prolonged which can cause tissue damage and loss of the function implicated in pathogenesis of a broad spectrum of human disorders. There is growing body of evidence indicating that chronic inflammation results from the failure in the resolution of inflammation (Nathan and Ding, 2010).

Upon inflammatory insult, tissue resident macrophages produce vasoactive and chemotactic mediators, which increase vascular permeability and promote the infiltration of leukocytes, especially neutrophils, into the inflamed site where they eliminate or neutralize invading molecules (Lee et al., 2013). During this process, neutrophils at the inflamed site undergo apoptosis, which are removed by macrophages. After engulfing apoptotic neutrophils, which is called efferocytosis, macrophages leave the inflamed tissue via the nearest lymph nodes. This terminates the inflammatory responses (Elliott et al., 2017; Lee et al., 2013). The resolution of inflammation is an active and dynamic process regulated by several endogenous anti-inflammatory and pro-resolving mediators of the innate immune system.

COX-2 is a rate-limiting enzyme in the arachidonic acid cascade responsible for the production of a distinct set of prostaglandins (PGs) in response to inflammatory stimuli. Non-steroidal anti-inflammatory drugs (NSAIDs), such as aspirin, have been used for the alleviation of inflammatory symptoms suppressing COX-2 activity/expression. Cancer preventive and therapeutic effects of some NSAIDs have been reported (Thun et al., 2002; Baron et al., 2003). However, pharmacological inhibition of COX-2 for the 
a

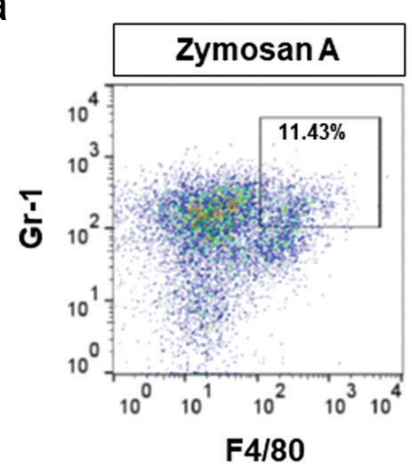

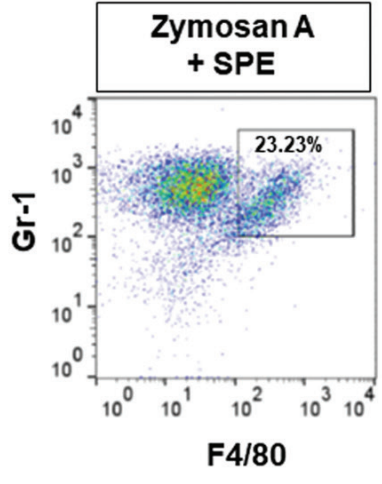

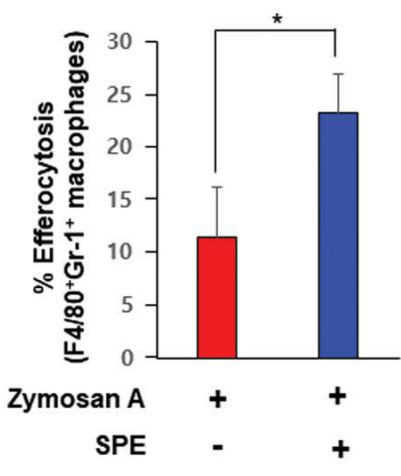

b<smiles>O=C(/C=C/c1ccc(O)c(O)c1)OC(Cc1ccc(O)c(O)c1)C(=O)O</smiles>
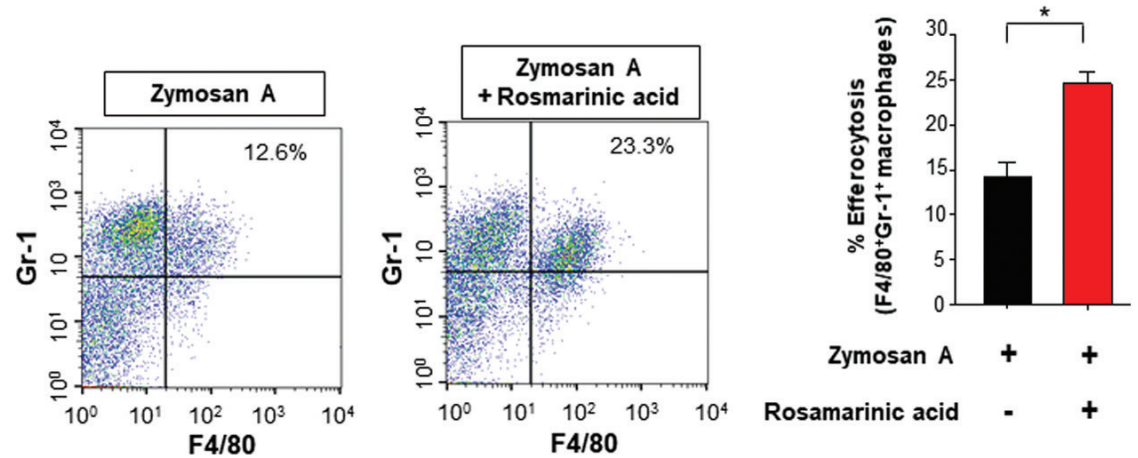

Zymosan A $+\quad+$

Rosamarinic acid - $\quad+$

C

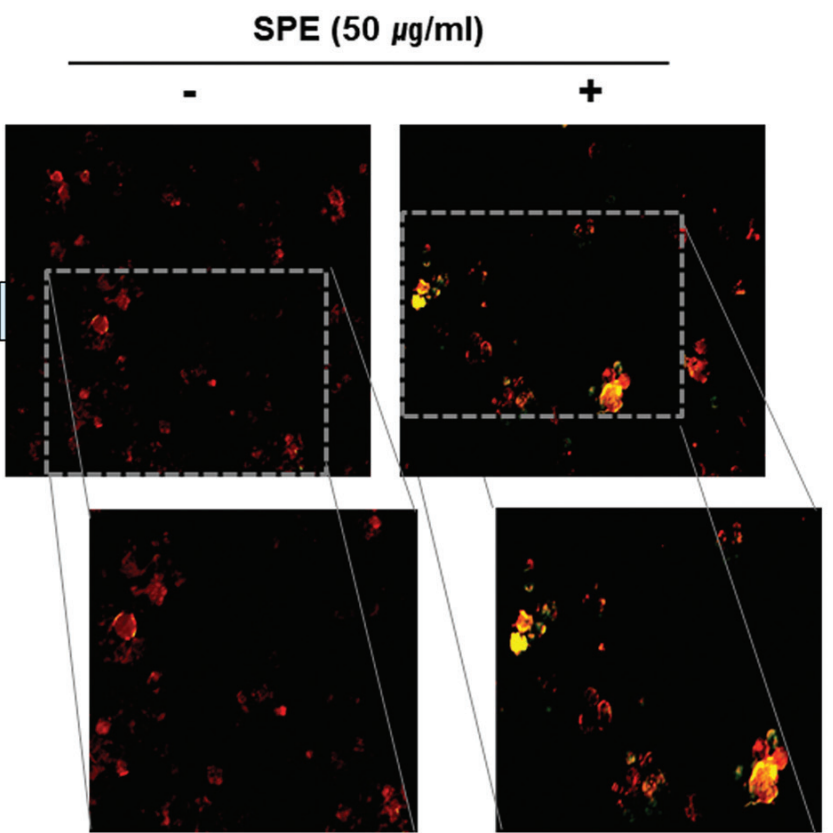

Figure 1. Potentiation of efferocytosis by the perilla extract and its pharmacologically active ingredient, rosmarinic acid in a zymosan A-induced murine peritonitis model. (a) Twelve $\mathrm{h}$ after intraperitoneal injection of zymosan A $(30 \mathrm{mg} / \mathrm{kg}), \mathrm{SPE}(100 \mathrm{mg} / \mathrm{kg})$ was given intraperitonally to C57BI/6 mice. Six hours later, peritoneal exudates were collected, and the proportion of macrophages engulfing neutrophils $\left(\mathrm{F} 4 / 80^{+} \mathrm{Gr}-1^{+}\right)$was measured by flow cytometry as described in Materials and methods. (b) Rosmarinic acid $(20 \mathrm{mg} / \mathrm{kg}$ ) was administered by intraperitoneal injection $12 \mathrm{~h}$ after intraperitoneal injection of zymosan A. The experimental conditions for analysis of efferocytosis are same as those described for (a). (c) The thioglycolate-elicited PMs treated with SPE $(50 \mathrm{\mu g} / \mathrm{ml})$ for $4 \mathrm{~h}$ were co-incubated with zymosan A-FITC (green) for an additional $1 \mathrm{~h}$. The phagocytic activity of PMs was analyzed by immunostaining using F4/80 antibody (red). The macrophages engulfing zymosan A was visualized under a fluorescence microscope. 
a
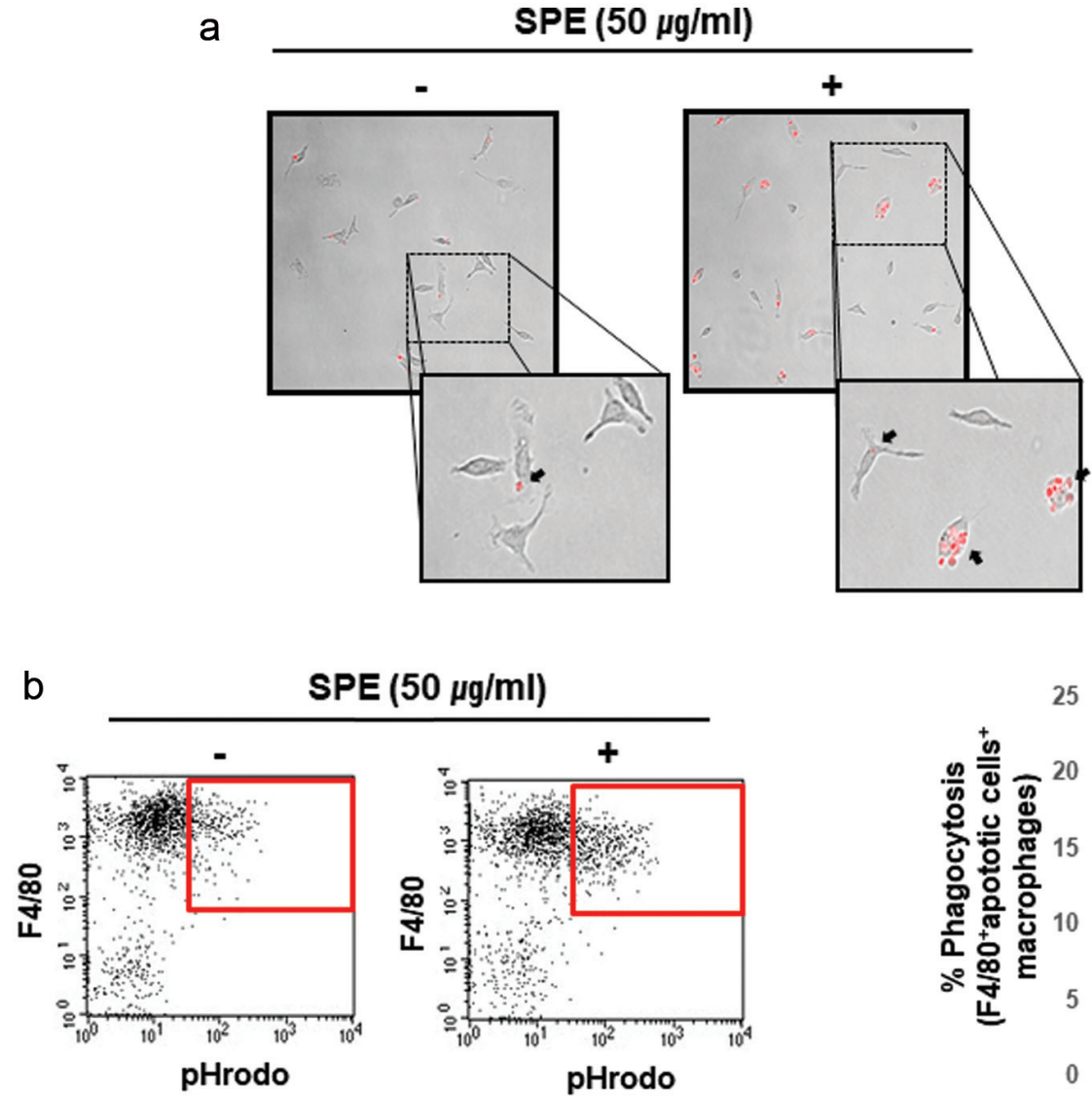

SPE $(50 \mu \mathrm{g} / \mathrm{ml})$
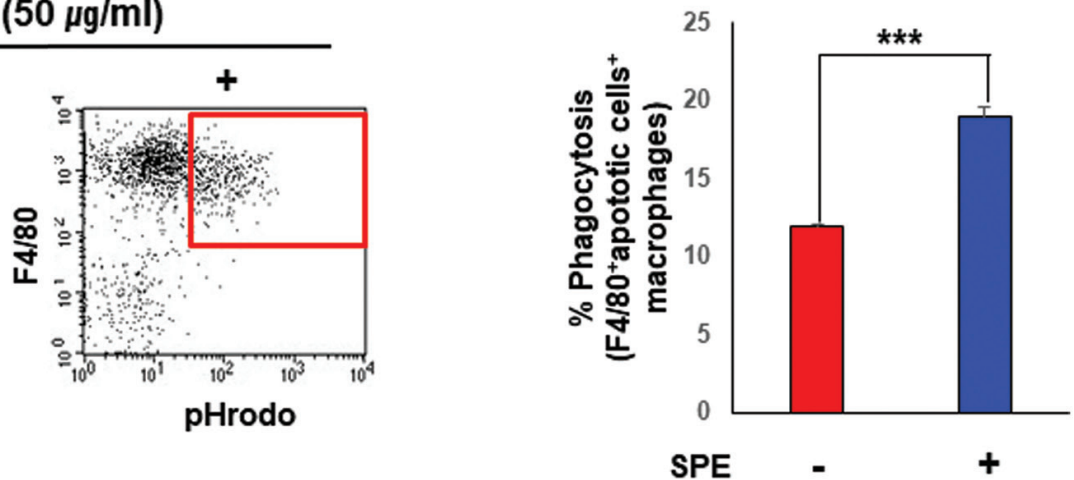

C

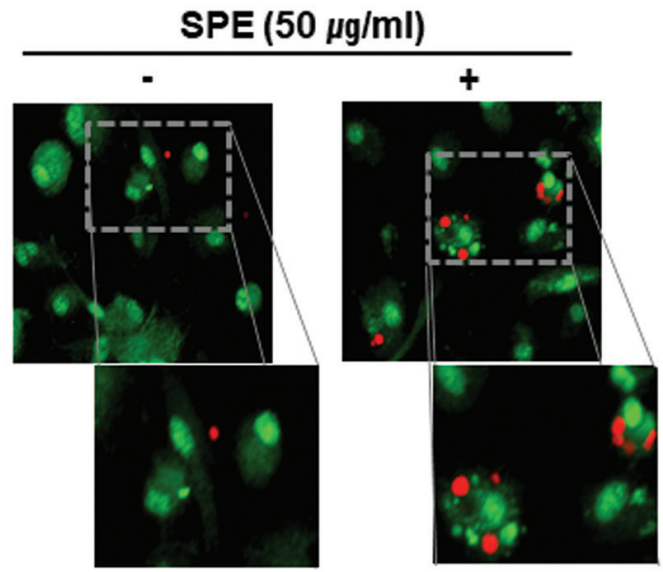

Green: F4/80 (macrophages)

Red: Apototic cells (thymocytes)

Figure 2. Enhancement of efferocytic activity of macrophages by the perilla extract. (a) The apoptotic thymocytes were prepared as described in Materials and methods. The apoptotic thymocytes labeled with a pH sensitive dye, pHrodo-SE were co-cultured with PMs pretreated for $4 \mathrm{~h}$ with SPE or vehicle. After incubation for $1 \mathrm{~h}$, the PMs were washed to remove free apoptotic cells, and subsequently subjected to microscopy. SPE pretreated PMs engulfed apoptotic thymocytes (red puncta) more actively. (b) Macrophages were differentiated from bone marrow cells. BMDMs treated with SPE (50 $\mu \mathrm{g} / \mathrm{ml}$ ) were co-incubated with pHrodo-labeled apoptotic thymocytes for $4 \mathrm{~h}$. The proportion of macrophages engulfing apoptotic cells was determined by flow cytometry. (c) To confirm the enhanced efferocytic ability of macrophages induced by SPE, BMDMs engulfing apoptotic cells were detected by immunostaining using F4/80 antibody (green; macrophage marker) and pHrodo-labeled apoptotic thymocytes (red). 


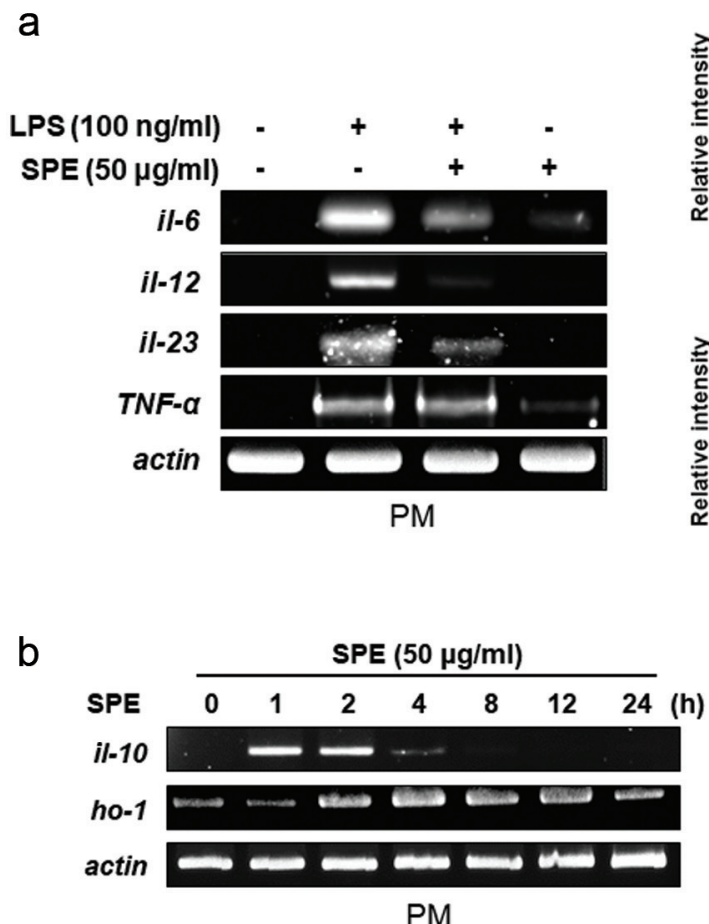

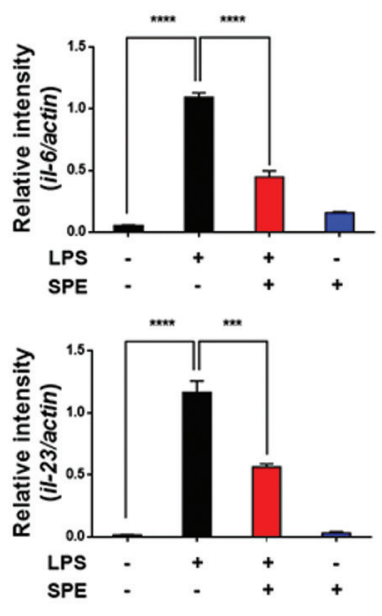
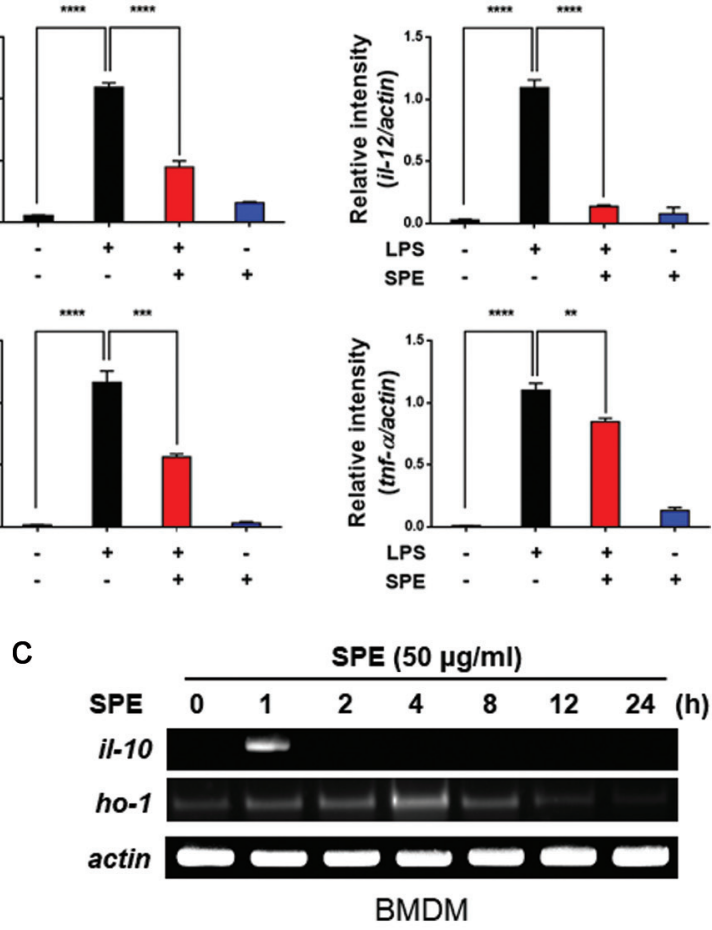

Figure 3. Inhibition of LPS-induced proinflammatory gene expression and induction of anti-inflammatory gene expression by perilla extract. (a) PMs isolated from C57BL/6 mice were treated with SPE $(50 \mu \mathrm{g} / \mathrm{ml})$ for $4 \mathrm{~h}$ followed by exposure to LPS (100 $\mathrm{ng} / \mathrm{ml})$ for additional $1 \mathrm{~h}$. The expression of proinflammatory cytokines in PMs was measured by RT-PCR as described in Materials and methods. Data are expressed as means \pm standard deviation. (b) PMs were treated with SPE (50 $\mathrm{gg} / \mathrm{ml})$ for the indicated time periods, and mRNA levels of IL-10, HO-1 and Nrf2 were determined by RT-PCR. Data are expressed as means \pm standard deviation. (c) After SPE (50 $\mathrm{gg} / \mathrm{ml})$ treatment of BMDMs, anti-inflammatory and antioxidant gene expression was determined by RT-PCR

treatment chronic inflammatory disorders is controversial (Wong, 2019) as COX-2 contributes not only to the proinflammatory process but also to the process leading to the resolution of inflammation (Luo et al., 2005; Park and Christman, 2006).

The so-called lipid mediator class switching spontaneously occurs during inflammatory responses in which $\mathrm{PGE}_{2}$, a principal proinflammatory product of $\mathrm{COX}-2$, activates the mechanism responsible for biosynthesis of anti-inflammatory and pro-resolving lipid mediators (Levy et al., 2001). Such elaborate lipid mediator class switching from proinflammatory to anti-inflammatory/pro-resolving status represents a critical event required for termination of inflammation (Lee et al., 2013). Thus, the administration of a COX-2 inhibitor may hamper the resolution of inflammation. Moreover, prolonged administration of NSAIDs can attenuate the cytoprotective functions of inflammatory cells, such as chemotaxis, bacterial killing, and phagocytosis. Therefore, mere suppression of inflammation may not be sufficient for the successful prevention of certain cancers. As many synthetic anti-inflammatory drugs have side effects and also hamper innate proresolving capacity, it is desirable to use safe natural products with proresolving as well as anti-inflammatory activities.

Perilla is a genus consisting of one major Asiatic crop species $P$. frutescens and a few wild species belonging to the mint family (Lamiaceae). P. frutescence has been used as an important traditional herbal medicine for treating various diseases including asthma, allergy, cough, chronic bronchitis, vomiting, depression, anxiety, and some intestinal disorders (Bachheti et al., 2014; Ahmed 2018). P. frutescence and some of its ingredients such as rosmarinic acid have been known to possess strong anti-inflammatory properties. We have previously reported that the SPE protects against experimentally induced colitis in mice and also inhibits activation of NF- $\mathrm{KB}$ and
STAT3, two major proinflammatory transcription factors, in cultured colon epithelial cells challenged with TNF- $\alpha$ (Park et al., 2017). We extended this study to the evaluation of proresolving effects of SPE. We found that SPE potentiated the efferocytic activity of PMs engulfing the apoptotic neutrophils in the zymosan A-induced murine peritonitis model and also engulfment of apoptotic cells by isolated macrophages including PMs and BMDMs. SPE also inhibited LPSinduced expression of proinflammatory signaling molecules while upregulating anti-inflammatory gene expression. By controlling the balance between proinflammatory and anti-inflammatory cytokines, SPE is likely to facilitate resolution of inflammation.

Engulfment of apoptotic cells by macrophages engages multiple sensing molecules that play important roles in recognizing dying cells and controlling phagocytosis. One of the best defined metabolic sensing molecules that links sensing of ingested apoptotic cells and the macrophage-mediated efferocytosis machinery is PPAR $\gamma$. (Elliott et al.). The roles for PPAR $\gamma$ and its ligands are emerging as they play an important role in all stages of the resolution of inflammation. Thus, PPAR $\gamma$ alters macrophage trafficking, induces neutrophil apoptosis and clearance, increases macrophage phagocytosis, and promotes alternative M2 macrophage activation (Croasdell et al., 2015). A PPAR $\gamma$ ligand, rosiglitazone increased M2 gene expression and enhanced efferocytosis of apoptotic neutrophils. Rosiglitazone and another PPAR $\gamma$ ligand pioglitazone attenuated airway neutrophilia in a corticosteroid-resistant mouse model of pulmonary inflammation (Lea et al., 2014).

In order to carry out the efferocytosis, the macrophages express surface scavenger receptors which recognize phosphatidyl serine exposed at the outer leaflet of the plasma membrane of apoptotic cells. One such scavenger receptor involved in efferocytosis is 
a

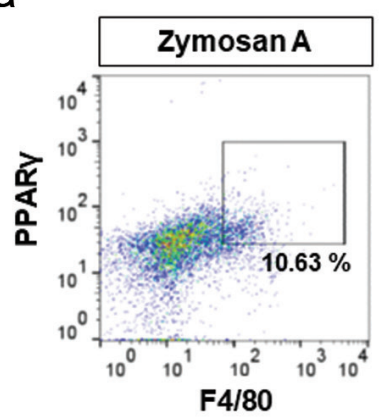

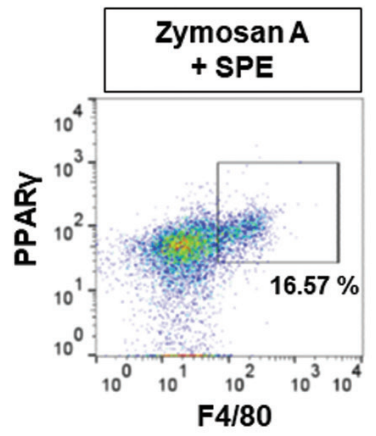

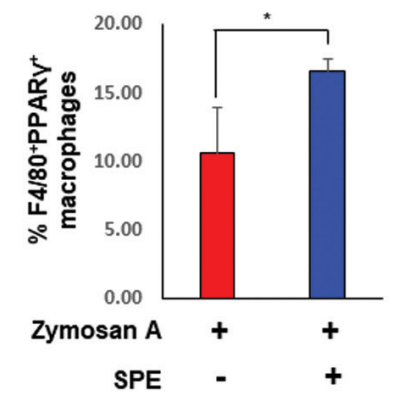

d

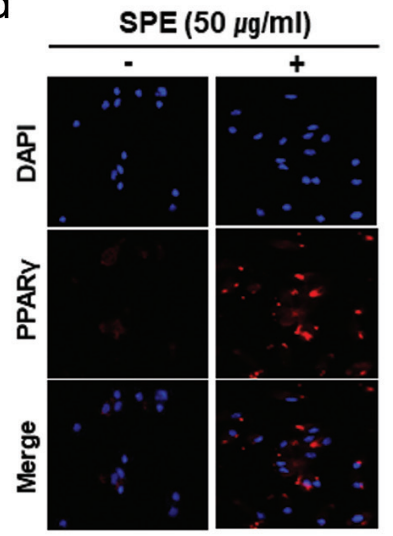

b

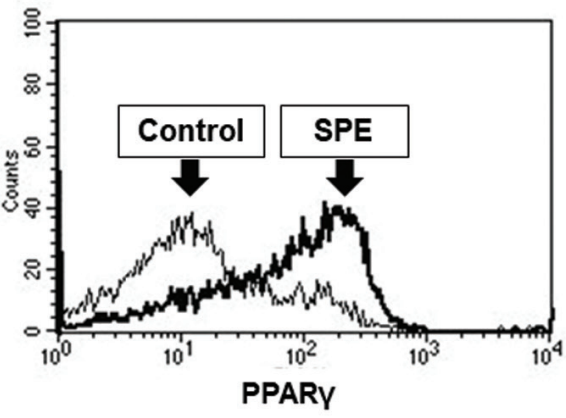

C

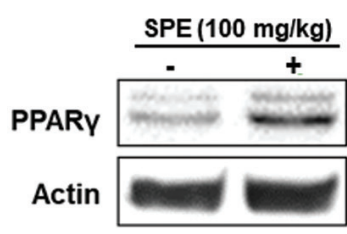

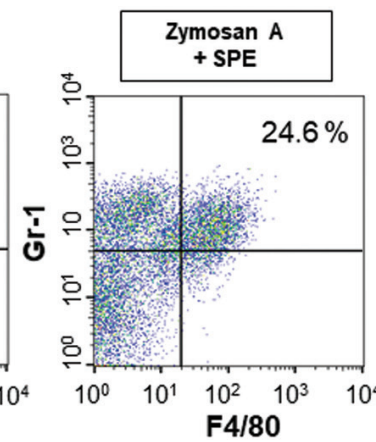

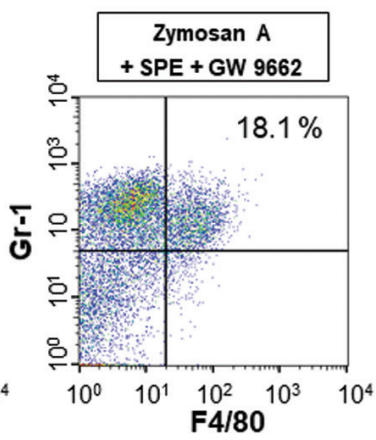

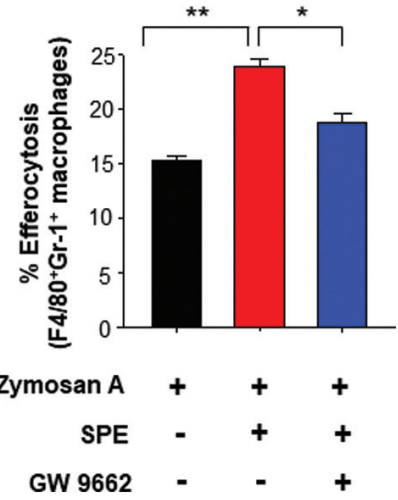

Figure 4. Role of PPARy in efferocytosis stimulated by perilla extract in the zymosan-induced murine peritonitis model. (a) The PPARy expression of PMs from mice was measured by flow cytometry. (b, c) Upregulation of PPARY expression by SPE in PMs. SPE (100 mg/kg) was co-injected into mouse peritoneal cavity with thioglycollate. After 4 days, the PMs were isolated and subjected to flow cytometry (b) and Western blot analysis (c). (d) The thioglycolate-elicited murine PMs were treated with PE $(50 \mathrm{\mu g} / \mathrm{ml})$ for $4 \mathrm{~h}$. The PPARy expression of macrophages was examined by immunofluorescence staining. (e) Mice injected with zymosan A (30 mg/kg) for $6 \mathrm{~h}$ were given a single intraperitoneal injection of vehicle or GW9662 (3 mg/kg) $1 \mathrm{~h}$ before SPE (100 mg/kg) treatment. The efferocytotic activity of macrophages was measured by flow cytometry.

CD36 that regulates the macrophage-mediated inflammatory response. The recognition of apoptotic neutrophils and their engulfment by macrophages then trigger secretion of anti-inflammatory cytokines like TGF- $\beta$ and IL-10 that dampen proinflammatory responses (Korns et al., 2011). CD36 expression in macrophages is mainly controlled by PPAR $\gamma$. Notably, inflammatory processes negatively regulate $\mathrm{CD} 36$ expression in macrophages, which is associated with a failure in the expression and activation of PPAR $\gamma$.

In inflammatory conditions, the anti-inflammatory transcription factor Nrf2 controls CD36 expression, independently of PPAR (Olagnier et al., 2011; Kim et al., 2017). The SPE-induced efferocytic activity of macrophages appears to be mediated through PPAR $\gamma$ activation as the pharmacologic inhibition of PPAR $\gamma$ attenuated the efferocytic activity of macrophages. Though not tested in macrophages, SPE administration has been shown to induce expression of Nrf2 and its target protein, HO-1 in mouse colon. We speculate that SPE-induced activation of PPAR $\gamma$ and/or Nrf2 may upregulate the CD36 expression in macrophages in mediating efferocytosis in vivo (Fig. 5). 

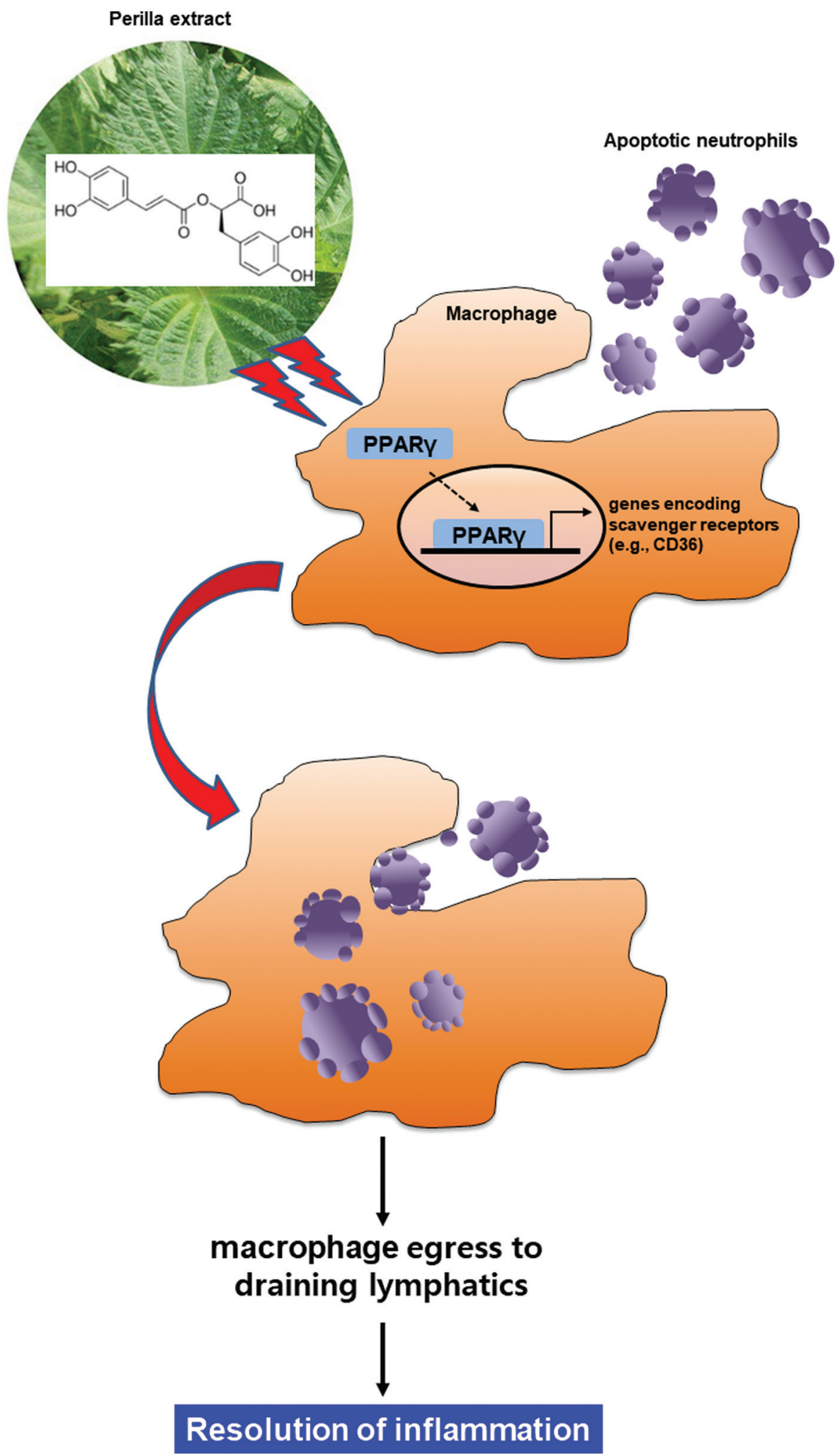

Figure 5. Proposed scheme illustrating the stimulatory effects of perilla extract and its ingredient rosmarinic acid on macrophage-mediated phagocytic removal of apoptotic neutrophils (efferocytosis). SPE activates the PPARy in macrophages, which upregulates the expression of CD36, a scavenger receptor involved in the recognition of phosphatidylserine epitopes exposed on the surface of apoptotic cells. This will facilitate the phagocytic removal of dying neutrophils, thereby completing the resolution of acute inflammation. 
Although the results are promising in preclinical studies, clinical data are insufficient, and further studies will be necessary to validate therapeutic effects of SPE and to ensure its safety.

\section{Acknowledgments}

This work was supported by the BK21 Plus Program (10Z2013 0000017) of the National Research Foundation of Korea and Amino Up Co. Ltd.

\section{References}

Ahmed, H.M. (2018). Ethnomedicinal, phytochemical and pharmacological investigations of Perilla frutescens (L.) Britt. Molecules 24: 102.

Asif, M. (2012). Phytochemical study of polyphenols in Perilla frutescens as an antioxidant. Avicenna J. Phytomed. 2: 169-78.

Bachheti, R.K., Joshi, A., and Ahmed, T. (2014). Phytopharmacological overview on Perilla frutescens. Int. J. Pharm. Sci., Rev. Res. 26: 55-61.

Bae, J.S., Han, M., Shin, H.S., Kim, M.-K., Shin, C.-Y., Lee, D.H., and Chung, J.H. (2017). Perilla frutescens leaves extract ameliorates ultraviolet radiation-induced extracellular matrix damage in human dermal fibroblasts and hairless mice skin. J. Ethnopharmacol. 195: 334-342.

Barnig, C., and Levy, B.D. (2015). Innate immunity is a key factor for the resolution of inflammation in asthma. Eur. Respir. Rev. 24: 141-153.

Baron, J.A., Cole, B.F., Sandler, R.S., Haile, R.W., Ahnen, D., Bresalier, R., McKeown-Eyssen, G., Summers, R.W., Rothstein, R., Burke, C.A., Snover, D.C., Church, T.R., Allen, J.I., Beach, M., Beck, G.J., Bond, J.H., Byers, T., Greenberg, E.R., Mandel, J.S., Marcon, N., Mott, L.A., Pearson, L., Saibil, F., and van Stolk, R.U. (2003). A randomized trial of aspirin to prevent colorectal adenomas. N. Engl. J. Med. 348: 891-899.

Boada-Romero, E., Martinez, Z., Heckmann, B.L., and Green, D.R. (2020). The clearance of dead cells by efferocytosis. Nat. Rev. Mol.Cell Biol. 21: 398-414.

Chen, M.L., Wu, C.H., Hung, L.S., and Lin, B.H. (2015). Ethanol extract of Perilla frutescenss suppresses allergen-specific Th2 responses and alleviates airway inflammation and hyperreactivity ovalbumin-sensitized murine model of asthma. Evid. Based Complement. Alternat. Med. 2015: 324265.

Croasdell, A., Duffney, P.F., Kim, N., Lacy, S.H., Sime, P.J., and Phipps, R.P. (2015). PPARy and the innate immune system mediate the resolution of inflammation. PPAR Res. 2015: 549691.

Elliott, M.R., Koster, K.M., and Murphy, P.S. (2017). Efferocytosis signaling in the regulation of macrophage inflammatory responses. J. Immunol. 198: 1387-1394.

Kim, W., Kim, H.U., Lee, H.N., Kim, S.H., Kim, C., Cha, Y.N., Joe, Y., Chung, H.T., Jang, J., Kim, K., Suh, Y.G., Jin, H.O., Lee, J.K., and Surh, Y.J. (2015). Taurine chloramine stimulates efferocytosis through upregulation of Nrf2-mediated heme oxygenase-1 expression in murine macrophages: Possible involvement of carbon monoxide. Antioxid. Redox Signal. 23: 163-177.

Kim, W., Lee, H.-N., Jang, J.-H., Kim, S.H., Lee, Y.-H., Hahn, Y.-I., Ngo, H.-K.-C., Choi, Y., Joe, Y., Chung, H.T., Chen, Y., Cha, Y.N., and Surh, Y.-J. (2017) 15-Deoxy- $\Delta^{12,14}$-prostaglandin $\mathrm{J}_{2}$ exerts proresolving effects through nuclear factor E2-related factor 2-induced expression of CD36 and heme oxygenase-1. Antioxid. Redox Signal. 10 27: 1412-1431.

Korns, D., Frasch, S.C., Fernandez-Boyanapalli, R., Henson, P.M., and Bratton, D.L. (2011). Modulation of macrophage efferocytosis in inflammation. Front. Immunol. 2: 57.

Kwak, Y., and Ju, J. (2015). Inhibitory activities of Perilla frutescens Britton leaf extract against the growth, migration, and adhesion of human cancer cells. Nutr. Res. Pract. 9: 11-16.

Lea, S., Plumb, J., Metcalfe, H., Spicer, D., Woodman, P., Fox, C., and Singh, D. (2014). The effect of peroxisome proliferator activated receptor- - ligands on in vitro and in vivo models of COPD. Eur. Respir. J. 43 409-420.

Lee, H.A., and Han, J.-S. (2012). Anti-inflammatory effect of Perilla frutescens (L.) Britton var. frutescens extract in LPS-stimulated RAW 264.7 macrophages. Prev. Nutr. Food Sci. 17: 109-115.

Lee, H.N., Na, H.K., and Surh, Y.-J. (2013). Resolution of inflammation as a novel chemopreventive strategy. Semin. Immunopathol. 35: 151161

Lee, H.N., and Surh, Y.-J. (2012). Therapeutic potential of resolvins in the prevention and treatment of inflammatory disorders. Biochem. Pharmacol. 84: 1340-1350.

Levy, B.D., Clish, C.B., Schmidt, B.K., and Serhan, C.N. (2001). Lipid mediator class switching during acute inflammation: signals in resolution. Nat. Immunol. 2: 612-619.

Lim, H.J., Woo, K.W., Lee, K.R., Lee, S.K., and Kim, H.P. (2014). Inhibition of proinflammatory cytokine generation in lung inflammation by the leaves of Perilla frutescens and its constituents. Biomol. Ther. (Seoul) 22: 62-67.

Luo, C., He, M.-L., and Bohlin, L. (2005). Is COX-2 a perpetrator or a protector? Selective COX-2 inhibitors remain controversial. Acta Pharmacologica Sin. 26: 926-933.

Makino, T., Furuta, A., Fujii, H., Nakagawa, T., Wakushima, H., Saito, K., and Kano, Y. (2001). Effect of oral treatment of Perilla frutescens and its constituents on type-I allergy in mice. Biol. Pharm. Bull. 24: 1206-1209.

Nathan, C., and Ding, A. (2010). Nonresolving inflammation. Cell 140: 871-882.

Olagnier, D., Lavergne, R.A., Meunier, E., Lefèvre, L., Dardenne, C., Aubouy, A., Benoit-Vical, F., Ryffel, B., Coste, A., Berry, A., and Pipy, B. (2011). Nrf2, a PPARy alternative pathway to promote CD36 expression on inflammatory macrophages: Implication for malaria. PLoS Pathog. 7: e1002254.

Ortega-Gómez, A., Perretti, M., and Soehnlein, O. (2013). Resolution of inflammation: an integrated view. EMBO Mol. Med. 5: 661-674.

Park, D.D., Yum, H.-W., Zhong, X., Kim, S.H., Kim, S.H., Kim, D.-H., Kim, S.-J., $\mathrm{Na}$, H.-K., Sato, A., Miura, T., and Surh, Y.-J. (2017). Perilla frutescens extracts protects against dextran sulfate sodium-induced murine colitis: NF-KB, STAT3, and Nrf2 as putative targets. Front. Pharmacol. 8: 412 .

Park, G.Y., and Christman, J.W. (2006). Involvement of cyclooxygenase-2 and prostaglandins in the molecular pathogenesis of inflammatory lung diseases. Am. J. Physiol. Lung Cell. Mol. Physiol. 290: L797-L805.

Soehnlein, O., and Lindbom, L. (2010). Phagocyte partnership during the onset and resolution of inflammation. Nat. Rev. Immunol. 10: 427439.

Thun, M.J., Henley, S.J., and Patrono, C. (2002). Nonsteroidal anti-inflammatory drugs as anticancer agents: mechanistic, pharmacologic, and clinical issues. J. Natl. Cancer Inst. 94: 252-266.

Ueda, H., and Yamazaki, M. (2001). Anti-inflammatory and anti-allergic actions by oral administration of a Perilla leaf extract in mice. Biosci. Biotech. Biochem. 65: 1673-1675.

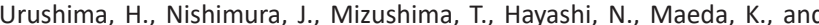
Ito, T. (2015). Perilla frutescens extract ameliorates DSS-induced colitis by suppressing proinflammatory cytokines and inducing antiinflammatory cytokines. Am. J. Physiol. Gastrointest. Liver Physiol. 308: G32-41.

Wang, X.F., Li, H., Jiang, K., Wang, Q.-Q, Zheng, Y.-H., Tang, W., and Tan, C.-H. (2018). Anti-inflammatory constituents from Perilla frutescens on lipopolysaccharide-stimulated RAW264.7 cells. Fitoterapia 130: 61-65.

Wong, R.S.Y. (2019). Role of nonsteroidal anti-inflammatory drugs (NSAIDs) in cancer prevention and cancer promotion. Adv. Pharmacol. Sci. 2019: 3418975 .

Yoon, Y.S., Kim, S.-Y., Kim, M.-J., Lim, J.-H., Cho, M.-S., and Kang, J.L. (2015) PPARY activation following apoptotic cell instillation promotes resolution of lung inflammation and fibrosis via regulation of efferocytosis and proresolving cytokines. Mucosal Immunol. 8: 1031-1046. 